\title{
Uso de metacaulim em concreto seco: uma abordagem por superfície de resposta
}

\author{
Use of metakaolin in no slump concrete: a response \\ surface approach
}

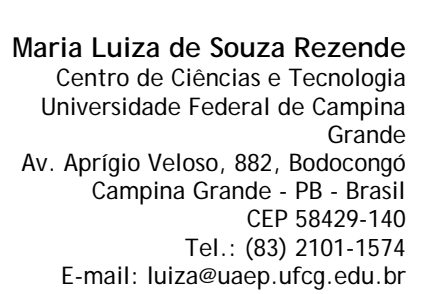

J osé Wallace Barbosa do Nascimento

Departamento de Engenharia Agrícola, Centro de Tecnologia e Recursos Naturais Universidade Federal de Campina Tel.: (83) 2101-1055 E-mail: wallace@deag.ufcg.edu.br

Gelmires de Araújo Neves Departamento de Engenharia de Materiais, Centro de Ciências e Tecnologia

Universidade Federal de Campina Grande Tel.: (83) 2101-1183

E-mail: gelmires@dema.ufcg.edu.br

Flávio Luiz Honorato da Silva

Departamento de Engenharia

Química, Centro de Tecnologia

Universidade Federal da Paraíba

Av. Aprígio Veloso, 882, Bodocongó Caixa-Postal 10087

Campina Grande - PB - Brasil CEP 58109725

Tel.: (83) 3216-7357

E-mail: flavioluizh@yahoo.com.br

Antônio Faria Leal

Departamento de Engenharia de Materiais, Centro de Tecnologia Universidade Federal da Paraíba Av. Aprígio Veloso, 882, Bodocongó Caixa-Postal 10087 Campina Grande - PB - Brasil CEP 58109725

Tel.: (83) 2101-1183

E-mail: leal@deag.ufcg.edu.br

Heber Carlos Ferreira Departamento de Engenharia de Materiais, Centro de Ciências e Tecnologia

Universidade Federal de Campina Grande

Tel.: (83) 3310-1183 Ramal 2 E-mail: heber@dema.ufcg.edu.br

Recebido em 21/07/11 Aceito em 26/05/12

\section{Maria Luiza de Souza Rezende J osé Wallace Barbosa do Nascimento Gelmires de Araújo Neves Flávio Luiz Honorato da Silva Antônio Farias Leal Heber Carlos Ferreira}

\section{Resumo}

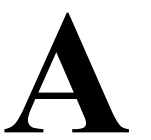
preocupação com o meio ambiente vem motivando diversas pesquisas sobre o uso de materiais pozolânicos em substituição parcial ao cimento em concretos e argamassas, entretanto poucos são os dados sobre tal uso em concretos destinados à produção de elementos prémoldados, concreto este que geralmente é denominado concreto seco ou sem abatimento, devido à baixa umidade requerida no processo de moldagem e prensagem. Este trabalho consistiu em um estudo preliminar sobre o uso de metacaulim em concreto seco, sendo analisados cinco traços de concreto com cinco teores de substituição em cinco tempos de cura, objetivando determinar quais os percentuais de substituição mais adequados, ou seja, aqueles que promovessem aumento na resistência à compressão simples e redução na absorção de água por imersão. Devido ao grande número de variáveis envolvidas, foi utilizado o planejamento experimental estatístico Delineamento Composto Central Rotacional (DCCR), sendo os resultados analisados pela Metodologia de Superfície de Resposta (MSR). A análise da superfície de resposta mostrou que, para todos os traços, os melhores resultados dentro dos parâmetros estabelecidos poderão ser encontrados com o uso de metacaulim em percentuais de $10 \%$ a $20 \%$ de substituição da massa do cimento aos 49 dias de cura.

Palavras-chave: Pozolana. Concreto. Concreto pré-moldado.

\section{Abstract}

The concern about the environment has motivated several studies on the use of pozzolanic materials as a partial replacement for cement in concrete and mortar. However, there are few data about such a use in concrete designed for the production of precast elements, which is usually called dry or no-slump concrete, due to the low humidity required in the process of molding and pressing. This paper reports a preliminary study on the use of metakaolin in no-slump concrete, analyzing five concrete mixtures with five levels of substitution, in five curing times, aiming to determine the most appropriate percentages of replacement, i.e., those that promote increase in compressive strength and reduction in water absorption by immersion. Due to the large number of variables involved, the statistical experimental design named Central Composite Rotational Design (CCRD) was used, and the results were analyzed using the Response Surface Methodology (RSM). The response surface analysis showed that for all concrete mixtures, the best results within the parameters established can be found with the use of metakaolin in percentages of $10 \%$ at $20 \%$ of replacement in the cement mass at 49 days of curing.

Keywords: Pozzolan. Concrete. Precast concrete. 


\section{Introdução}

O termo "concreto seco" é aplicado aos concretos cuja umidade geralmente varia entre $6 \%$ e $8 \%$ (FRASSON JUNIOR, 2000), umidade esta que faz com que eles possuam abatimento zero, o que justifica também a denominação de concreto sem abatimento ou no slump. Tais concretos são utilizados na indústria de pré-moldados e de concretos compactados, em que a retirada do ar aprisionado é feita por máquinas vibroprensas ou rolo compactador, para que as misturas atinjam a compactação necessária para alcançar as propriedades mecânicas desejadas.

A alvenaria que utiliza blocos de concreto vem-se mostrando uma ótima solução construtiva, sendo largamente utilizada tanto em obras residenciais como em galpões industriais, principalmente nesses últimos, em que a rapidez na execução da obra representa um dos fatores mais importantes em um empreendimento. Comparando-se à adoção de tijolos cerâmicos, o uso de blocos de concreto em alvenarias pode promover uma redução de $30 \%$ no custo da obra.

Em razão da crescente demanda, a indústria de pré-moldados de concreto apresenta índices de crescimento bastante significativos, sendo os blocos de vedação (12,32\%), blocos estruturais $(12,31 \%)$ e pisos intertravados $(12,31 \%)$ os produtos de cimento que tiveram maior crescimento em 2010 (SINDICATO..., 2011).

Ainda segundo dados do Sindicato Nacional das Indústrias de Produtos de Cimento (Sinaprocim), houve em 2009 decréscimos de 2,2\% no consumo de cimento e aumento de 3,3\% no consumo de adições minerais. Essa tendência é justificada pelo fato de que, pela necessidade de desforma imediata, o cimento geralmente utilizado nas indústrias de pré-moldados exige altas resistências iniciais, sendo o CPV-ARI (ABNT, 1991a) o mais indicado (FERNANDES, 2008). Constituído por 95\% de clínquer, o cimento CPV-ARI pode ser equiparado ao CEM I 52,5R, citado por Cassagnabère et al. (2009) como um dos tipos de cimento de maior custo ambiental e energético.

$\mathrm{O}$ uso de adições minerais que possuam propriedades pozolânicas tais como sílica ativa, cinzas volantes, metacaulim e cinzas de casca de arroz em substituição parcial ao cimento é uma alternativa para reduzir tais custos, podendo promover ainda melhorias significativas nas propriedades mecânicas de argamassas e concreto.

O princípio básico que justifica tais melhorias consiste no fato de que, para que um material pozolânico possa desenvolver propriedades cimentícias, há a necessidade da presença de hidróxido de cálcio $(\mathrm{CH})$ e água. De acordo com Sabir, Wild e Bai (2001), o cimento Portland, se totalmente hidratado, produz $\mathrm{CH}$ em cerca de $28 \%$ de seu próprio peso, e essa produção não interfere nas propriedades mecânicas, porém o hidróxido de cálcio excedente reage com a pozolana adicionada resultando em silicatos de cálcio hidratados adicionais que proporcionam melhorias em tais propriedades.

Entre os materiais pozolânicos, o metacaulim é o material pozolânico resultante da calcinação de argilas cauliníticas a temperaturas que variam entre $500{ }^{\circ} \mathrm{C}$ e $800{ }^{\circ} \mathrm{C}$ (SOUZA; DAL MOLIN, 2002), dependendo da composição da argila utilizada. Foi considerado por Cassagnabère et al. (2009), após comparar o desempenho de quatro tipos de metacaulim com fillers calcários e silicosos, e sílica ativa, uma solução muito promissora na substituição do cimento Portland em concretos destinados à produção de elementos pré-moldados. Entretanto, a grande maioria dos estudos sobre a influência do metacaulim em concretos aborda o uso em concretos plásticos (VU; STROEVEN; BUI, 2001; GRUBER et al., 2001; SABIR; WILD; BAI, 2001; SOUZA, 2003; AL-AKHRAS, 2006; ROSSIGNOLO; OLIVEIRA, 2006; CARMO; PORTELLA, 2008; SIDDIQUE; KLAUS, 2009), sendo encontradas, na literatura pesquisada, poucas pesquisas que objetivam a avaliação da influência do metacaulim nas propriedades mecânicas do concreto com baixo teor de umidade (concreto seco), entre elas Pettermann (2006) e Cassagnabère et al. (2009).

A influência do metacaulim depende, além de sua pureza e reatividade, do teor de cimento no concreto e do período e tipo de cura; logo, torna-se bastante extensa a análise experimental da influência de todas as variáveis envolvidas. O planejamento experimental fatorial permite escolher o método estatístico adequado para que as observações (ensaios) sejam feitas em pontos específicos do espaço amostral, não alterando o comportamento das variáveis de resposta e obtendo-se, assim, informações relevantes com um número menor de corpos de prova. Segundo Rodrigues e Iemma (2009), se validado estatisticamente o modelo matemático elaborado através deste método, as condições otimizadas poderão ser determinadas através da análise da superfície de resposta obtida.

A Metodologia de Superfície de Resposta (MSR), fundamentada na modelagem matemática de respostas, permite identificar a influência entre as variáveis independentes (fatores controlados) e as dependentes (respostas) do sistema analisado. Sua 
utilização é indicada quando se deseja otimizar as variáveis analisadas visando obter as condições ótimas que venham a maximizar uma reação, sendo considerado por Gunawan et al. (2005) um método estatístico eficaz para esse fim.

Objetivou-se, então, com este trabalho determinar mediante a análise por superfície de resposta quais os traços, teores de substituição e tempos de cura mais apropriados para a utilização de metacaulim em substituição ao cimento em concretos destinados à produção de blocos pré-moldados para alvenaria.

\section{Materiais e métodos}

Neste trabalho foram utilizados os seguintes materiais: cimento CPV-ARI (fabricante Cimpor Brasil), Metacaulim HP (fabricante Metacaulim do Brasil Ltda.) e, como agregados, brita 0 - NBR 7211 (ABNT, 2009), areia de granulometria média (ABNT, 2009) de natureza quartzosa, oriunda do rio Paraíba, e pó de pedra. As características físicas, químicas e mecânicas do cimento e do metacaulim foram fornecidas pelos fabricantes e estão descritas nas Tabelas 1 e 2.

Os dados para a caracterização física da brita, areia e pó de pedra estão descritos na Tabela 3.

A composição dos agregados foi baseada no menor índice de vazios, visando alcançar maiores massas unitárias, as quais são diretamente proporcionais à resistência à compressão simples: $37 \%$ de brita, $40 \%$ de areia e $23 \%$ de pó de pedra, variando-se a quantidade de aglomerante. A relação $\mathrm{A} /(\mathrm{C}+\mathrm{A})$, ou seja, água/(cimento+adição) variou entre $8 \%$ e $9 \%$ para as composições com maior teor de aglomerante, ou seja, maior quantidade de finos. A massa necessária para cada corpo de prova foi calculada tomando-se por base a massa específica de blocos recém-moldados por vibroprensagem na JAR Indústria de Premoldados Ltda. e, a partir dela, a quantidade de golpes necessária para a obtenção do volume referente aos corpos de prova.

Tabela 1 - Características físicas, químicas e mecânicas do cimento CPV-ARI

\begin{tabular}{|c|c|c|c|c|c|c|c|}
\hline & \multicolumn{5}{|c|}{ Deterninações } & \multicolumn{2}{|c|}{ Resultados } \\
\hline Físicas & $\begin{array}{l}\text { Área específica (BE } \\
\text { Finura na malha \#20 } \\
\text { Massa específica NB } \\
\text { Tempo de pega } \\
\text { NBR NM } 65 \text { (ABNT }\end{array}$ & $\begin{array}{l}\text { R } 1157 \\
\text { M } 23(A \\
\text { O2) }\end{array}$ & $\begin{array}{l}\text { BNT, } 1 \\
\text { T, } 2000 \\
\end{array}$ & $\begin{array}{l}\text { 1b) } \\
\text { Início d } \\
\text { Fim da }\end{array}$ & & $\begin{array}{l}17.000 \\
0,25 \% \\
3,14 \mathrm{~g} / \mathrm{c} \\
3 \mathrm{~h} 00 \mathrm{mi} \\
4 \mathrm{~h} 10 \mathrm{mi}\end{array}$ & \\
\hline $\begin{array}{l}\text { Mecânicas } \\
\text { (MPa) }\end{array}$ & $\begin{array}{l}\text { Resistência à compr } \\
\text { NBR } 7215 \text { (ABNT, }\end{array}$ & & & $\begin{array}{l}1^{\circ} \text { dia } \\
2^{\circ} \text { dia } \\
3^{\circ} \text { dia }\end{array}$ & & $\begin{array}{l}24,4 \\
33,8 \\
38,3\end{array}$ & \\
\hline $\begin{array}{l}\text { Químicas } \\
(\%)\end{array}$ & $\begin{array}{cc}\mathrm{SiO}_{2} & \mathrm{Al}_{2} \mathrm{O}_{3} \\
18,1 & 4,4 \\
\text { Resíduo insolúvel } \\
\text { Perda ao fogo }\end{array}$ & $\begin{array}{c}\mathrm{Fe}_{2} \mathrm{O}_{3} \\
2,8\end{array}$ & $\begin{array}{c}\mathrm{Na}_{2} \mathrm{O} \\
0,03\end{array}$ & $\begin{array}{c}\mathrm{K}_{2} \mathrm{O} \\
1,4\end{array}$ & $\begin{array}{l}\mathrm{CaO} \\
60,4\end{array}$ & $\begin{array}{c}\mathrm{MgO} \\
6,1 \\
0\end{array}$ & $\begin{array}{c}\mathrm{SO}_{3} \\
3,2\end{array}$ \\
\hline
\end{tabular}

Tabela 2 - Características físicas e químicas do metacaulim HP

\begin{tabular}{|c|c|c|c|c|c|c|}
\hline & \multicolumn{5}{|c|}{ Determinações } & Resultados \\
\hline Físicas & \multicolumn{5}{|c|}{$\begin{array}{l}\text { Área específica BET } \\
\text { Umidade } \\
\text { Diâmetro médio das partículas } \\
\text { Massa específica }\end{array}$} & $\begin{array}{c}327.000 \mathrm{~cm}^{2} / \mathrm{g} \\
0,6 \% \\
12,4 \mu \mathrm{m} \\
2,65 \mathrm{~g} / \mathrm{cm}^{3}\end{array}$ \\
\hline $\begin{array}{l}\text { Fases } \\
\text { cristalinas } \\
(\text { DRX) }\end{array}$ & \multicolumn{4}{|c|}{$\begin{array}{l}\mathrm{SiO}_{2} \\
\mathrm{Al}_{2} \mathrm{Si}_{2} \mathrm{O}_{5}(\mathrm{OH})_{4} \\
\mathrm{KMg}_{3} \mathrm{Si}_{3} \mathrm{AlO}_{10}(\mathrm{FeOH})_{2} \\
\mathrm{Fe}_{2} \mathrm{O}_{3} \\
\mathrm{Fe}_{2} \mathrm{TiO}_{3}\end{array}$} & $\begin{array}{l}\text { Sílica } \\
\text { Caulinita } \\
\text { Flogopita } \\
\text { Hematita } \\
\text { Ilmenita }\end{array}$ & $\begin{array}{l}\text { Quartzo } \\
\text { Caulinita } \\
\text { Flogopita } \\
\text { Hematita } \\
\text { Ilmenita }\end{array}$ \\
\hline $\begin{array}{l}\text { IAP - } \\
\text { Chapelle } \\
\text { modificado }\end{array}$ & \multicolumn{5}{|c|}{ Atividade pozolânica a $90 \pm 5^{\circ} \mathrm{C}$ (mg CaO/g amostra) } & 771,2 \\
\hline \multirow{2}{*}{$\begin{array}{l}\text { Químicas } \\
(\%)\end{array}$} & $\begin{array}{l}\mathrm{SiO}_{2} \\
51,57\end{array}$ & $\begin{array}{c}\mathrm{Al}_{2} \mathrm{O}_{3} \\
40,5\end{array}$ & $\begin{array}{c}\mathrm{Fe}_{2} \mathrm{O}_{3} \\
2,8\end{array}$ & $\begin{array}{c}\mathrm{Na}_{2} \mathrm{O} \\
0,08\end{array}$ & $\begin{array}{l}\mathrm{K}_{2} \mathrm{O} \\
0,18\end{array}$ & $\begin{array}{c}\mathrm{SiO}_{2}+\mathrm{Al}_{2} \mathrm{O}_{3}+\mathrm{Fe}_{2} \mathrm{O}_{3} \\
94,87\end{array}$ \\
\hline & \multicolumn{5}{|c|}{ Perda ao fogo } & 2,62 \\
\hline
\end{tabular}


Tabela 3 - Características físicas do agregado graúdo (brita 0), areia e pó de pedra

\begin{tabular}{l|c|c|c}
\hline \multicolumn{1}{c|}{ Determinações } & \multicolumn{3}{c}{ Resultados obtidos } \\
\cline { 2 - 4 } & Brita & Areia & Pó de pedra \\
\hline DMC (mm) NBR NM 248 (ABNT, 2003b) & 9,5 & 4,8 & 2,4 \\
Módulo de finura NBR NM 248 (ABNT, 2003b) & 5,99 & 3,58 & 3,05 \\
Massa unitária (g/cm³) NBR NM 45 (ABNT, 2006) & 1,54 & 1,46 & 1,43 \\
Massa específica (g/cm ${ }^{3}$ ) NBR NM 53 (ABNT, 2003a) & 2,674 & 2,591 & 2,688 \\
\hline
\end{tabular}

Tabela 4 - Quantitativos de material (p/1 corpo de prova) dos traços utilizados

\begin{tabular}{|c|c|c|c|c|c|c|c|c|c|c|}
\hline \multirow[b]{2}{*}{ Traço } & \multirow[b]{2}{*}{ Ensaio } & \multicolumn{2}{|c|}{ Cimento } & \multicolumn{2}{|c|}{ Metacaulim } & \multirow[b]{2}{*}{$\begin{array}{c}\text { Brita } \\
\text { (g) }\end{array}$} & \multirow[b]{2}{*}{$\begin{array}{c}\text { Areia } \\
\text { (g) }\end{array}$} & \multirow{2}{*}{$\begin{array}{c}\text { Pó de } \\
\text { pedra } \\
\text { (g) }\end{array}$} & \multirow[b]{2}{*}{$\begin{array}{c}\text { Água } \\
\text { (g) }\end{array}$} & \multirow{2}{*}{$\begin{array}{c}\text { Massa } \\
\text { total } \\
(\mathrm{g}) \\
\end{array}$} \\
\hline & & $\begin{array}{c}\text { TC } \\
(\%) \\
\end{array}$ & (g) & $\begin{array}{c}\text { TS } \\
(\%) \\
\end{array}$ & (g) & & & & & \\
\hline \multirow{2}{*}{$1: 3,83$} & Ref. & 26 & 87 & 0 & 0 & 123 & 133 & 77 & 38 & 458 \\
\hline & 14 & & 69,60 & 20 & 17,40 & 123 & 133 & 77 & 38 & 458 \\
\hline \multirow{3}{*}{$1: 4,25$} & Ref. & 24 & 80 & 0 & 0 & 126 & 136 & 78 & 38 & 458 \\
\hline & 5,7 & & 68,80 & 14 & 11,20 & 126 & 136 & 78 & 38 & 458 \\
\hline & 6,8 & & 59,20 & 26 & 20,80 & 126 & 136 & 78 & 38 & 458 \\
\hline \multirow{4}{*}{$1: 5,15$} & Ref. & 20 & 69 & 0 & 0 & 131 & 142 & 82 & 34 & 458 \\
\hline & 9 & & 62,10 & 10 & 6,90 & 131 & 142 & 82 & 34 & 458 \\
\hline & $\begin{array}{l}11,12, \\
15 \text { a } 17\end{array}$ & & 55,20 & 20 & 13,80 & 131 & 142 & 82 & 34 & 458 \\
\hline & 10 & & 48,30 & 30 & 20,70 & 131 & 142 & 82 & 34 & 458 \\
\hline \multirow{3}{*}{$1: 6,44$} & Ref. & 16 & 57 & 0 & 0 & 136 & 147 & 84 & 34 & 458 \\
\hline & 1,3 & & 49,02 & 14 & 7,98 & 136 & 147 & 84 & 34 & 458 \\
\hline & 2,4 & & 42,18 & 26 & 14,82 & 136 & 147 & 84 & 34 & 458 \\
\hline \multirow{2}{*}{$1: 7,67$} & Ref. & 14 & 49 & 0 & 0 & 139 & 151 & 86 & 33 & 458 \\
\hline & 13 & & 39,20 & 20 & 9,80 & 139 & 151 & 86 & 33 & 458 \\
\hline
\end{tabular}

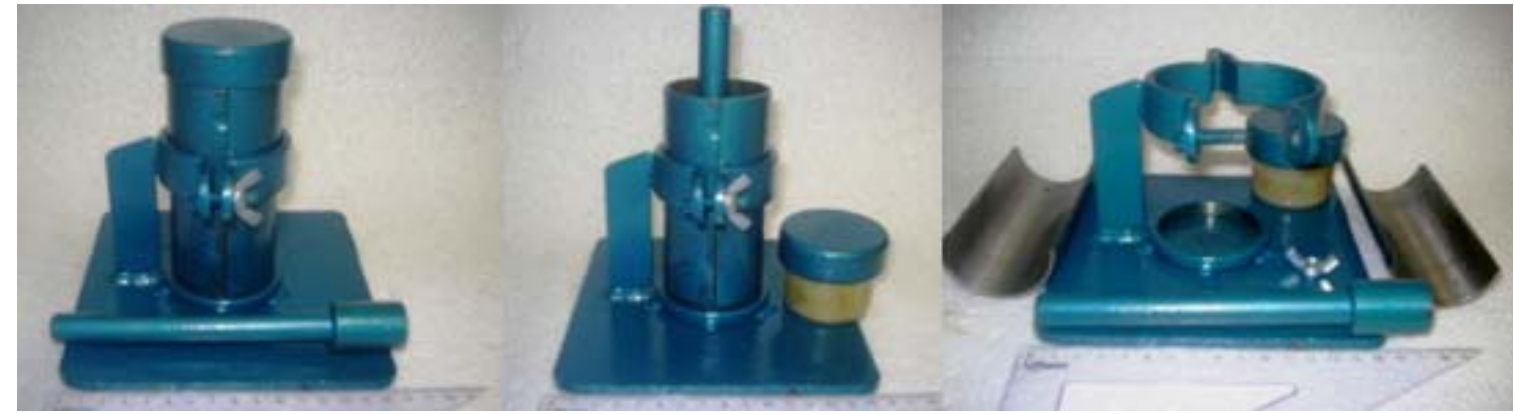

Figura 1 - Moldes bipartidos utilizados para a confecção dos corpos de prova

A substituição do cimento por metacaulim foi analisada em percentuais de 10\%, 14\%, 20\%, 26\% e $30 \%$ da massa do cimento, sendo os resultados também comparados com os encontrados para concretos de referência, ou seja, sem metacaulim. Os percentuais de substituição analisados foram escolhidos com base no fato de que o limite de hidróxido de sódio liberado pelo cimento, se totalmente hidratado, é de $28 \%$ da massa do cimento. A Tabela 4 descreve os traços (relação aglomerante/agregado), em massa, dos concretos utilizados neste trabalho, assim como as quantidades de material utilizado para moldagem de um corpo de prova.
Para a moldagem, foram utilizados moldes bipartidos, adaptados do modelo sugerido por Frasson Junior (2000), os quais permitem a desmoldagem imediata (Figura 1).

Após a homogeneização em misturador mecânico, a massa referente a cada corpo de prova foi colocada no molde em quatro camadas, sendo aplicados em cada uma 20 golpes utilizando-se um soquete semelhante ao recomendado pela NBR 7215 (ABNT, 1996). O acabamento e a compactação final foram obtidos encaixando-se o tarugo de nylon, o qual, após comprimido com golpes de martelo de borracha, limita a altura do corpo de prova em 100 mm (Figura 2). 


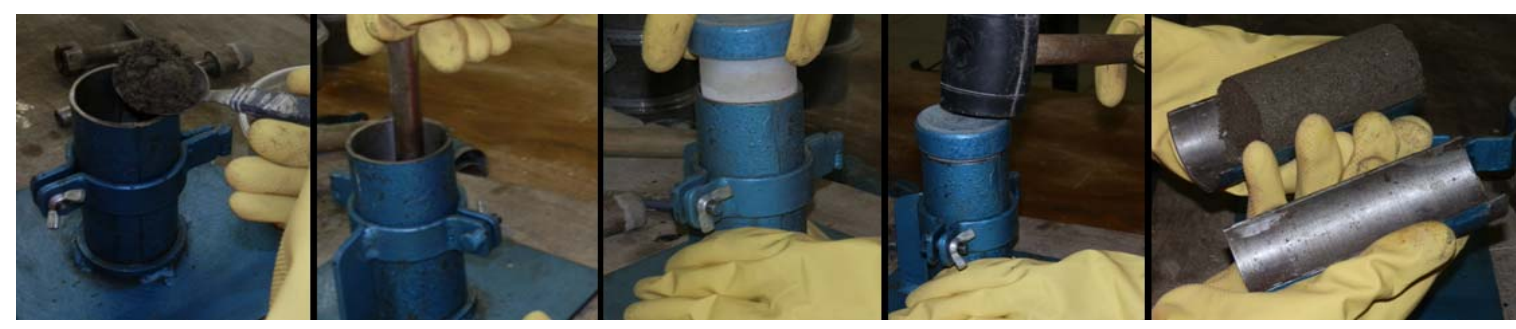

Figura 2 - Sequência de etapas para moldagem dos corpos de prova

Tabela 5 - Valores reais e codificados das variáveis independentes

\begin{tabular}{lccccc}
\hline \multirow{2}{*}{ Variáveis independentes } & \multicolumn{5}{c}{ Níveis } \\
\cline { 2 - 6 } & $\mathbf{- \alpha ( \mathbf { ( 1 , 6 8 ) }}$ & $\mathbf{- 1}$ & $\mathbf{0}$ & $\mathbf{+ 1}$ & $+\boldsymbol{+ \alpha}(+\mathbf{1 , 6 8 )}$ \\
\hline Teor de cimento - TC (\%) & 14 & 16 & 20 & 24 & 26 \\
Teor de substituição - TS (\%) & 10 & 14 & 20 & 26 & 30 \\
Tempo de cura - C (dias) & 7 & 24 & 49 & 74 & 91 \\
Teor de cimento - TC (\%) & 14 & 16 & 20 & 24 & 26 \\
\hline
\end{tabular}

Tabela 6 - Matriz Delineamento Composto Central Rotacional (DCCR)

\begin{tabular}{ccccccc}
\hline \multirow{2}{*}{ Ensaios } & \multirow{2}{*}{$\mathbf{X}_{\mathbf{1}}$} & \multirow{2}{*}{$\mathbf{X}_{\mathbf{2}}$} & \multirow{2}{*}{$\mathbf{X}_{\mathbf{3}}$} & \multicolumn{3}{c}{ Valores codificados } \\
\cline { 5 - 7 } & & & & $\mathbf{T S}(\%)$ & $\mathbf{C}(\mathbf{d i a s})$ & $\mathbf{T C}(\mathbf{\%})$ \\
\hline 1 & -1 & -1 & -1 & 14 & 24 & 16 \\
2 & +1 & -1 & -1 & 26 & 24 & 16 \\
3 & -1 & +1 & -1 & 14 & 74 & 16 \\
4 & +1 & +1 & -1 & 26 & 74 & 16 \\
5 & -1 & -1 & +1 & 14 & 24 & 24 \\
6 & +1 & -1 & +1 & 26 & 24 & 24 \\
7 & -1 & +1 & +1 & 14 & 74 & 24 \\
8 & +1 & +1 & +1 & 26 & 74 & 24 \\
9 & $-1,68$ & 0 & 0 & 10 & 49 & 20 \\
10 & $+1,68$ & 0 & 0 & 30 & 49 & 20 \\
11 & 0 & $-1,68$ & 0 & 20 & 7 & 20 \\
12 & 0 & $+1,68$ & 0 & 20 & 91 & 20 \\
13 & 0 & 0 & $-1,68$ & 20 & 49 & 14 \\
14 & 0 & 0 & $+1,68$ & 20 & 49 & 26 \\
15 & 0 & 0 & 0 & 20 & 49 & 20 \\
16 & 0 & 0 & 0 & 20 & 49 & 20 \\
17 & 0 & 0 & 0 & 20 & 49 & 20 \\
\hline
\end{tabular}

As primeiras 24 h de cura foram em câmara úmida (95\% umidade, $25^{\circ} \mathrm{C}$ ), sendo depois embalados individualmente em sacos plásticos fechados hermeticamente permanecendo em cura ambiente até a data dos ensaios, os quais foram executados aos 7, 24, 49, 74 e 91 dias após a moldagem. Foram confeccionados cinco corpos de prova para cada ensaio, sendo desprezados os resultados extremos, ou seja, os valores máximos e mínimos de cada experimento. Avaliou-se a resistência à compressão simples e a absorção de água por imersão, por entender-se que são as propriedades mais representativas das reações ocorridas durante a cura do concreto, sendo os ensaios realizados em conformidade com a norma NBR 12118 (ABNT, 2010).
Foi utilizado o planejamento experimental estatístico Delineamento Composto Central Rotacional (DCCR), que compreende um fatorial $2^{3}$, incluindo seis pontos axiais e três pontos centrais, perfazendo um total de 17 experimentos. A denominação “Central Rotacional” origina-se do fato de que todos os pontos escolhidos no espaço amostral são equidistantes do ponto central. O planejamento fatorial e os valores reais e codificados das variáveis independentes estudadas estão apresentados na Tabela 5.

Na Tabela 6 está apresentada a matriz que descreve os experimentos.

O planejamento experimental foi executado com três repetições e analisado estatisticamente 
utilizando-se o programa Statistica 5.0, da StatSoft, em um nível de confiança de 95\%. As médias obtidas dos três resultados intermediários foram também comparadas com as médias dos resultados obtidos para cada ensaio dos corpos de prova de referência, ou seja, sem metacaulim.

\section{Resultados e discussão}

A Tabela 7 apresenta os valores (respostas) dos parâmetros de resistência à compressão simples (RC) e absorção de água (AA) dos corpos de prova após os respectivos períodos de cura.

A Figura 3 representa esquematicamente as médias dos resultados para resistência à compressão, comparadas com as médias obtidas para corpos de prova de referência.

Observando-se a Figura 3, pode-se constatar que o ensaio 6, correspondente ao traço 1:4,25 com $26 \%$ de metacaulim aos 24 dias de cura, apresentou acréscimo de $16 \%$ na resistência à compressão em relação ao traço de referência, conflitando com o resultado encontrado para o traço 1:3,83 (ensaio 14), cuja substituição do cimento por $20 \%$ de metacaulim acarretou uma redução em $16 \%$ da resistência à compressão aos 49 dias de cura. Provavelmente, o teor de umidade para o traço 1:3,83 foi insuficiente, reduzindo a hidratação do cimento e, por conseguinte, a quantidade de hidróxido de sódio liberada, o que prejudicou a atividade pozolânica pela incorporação do metacaulim. Pozolanas em excesso nos concretos podem ser prejudiciais devido à redução de aglomerante, como pode ser notado no ensaio 10 (traço 1:5,15 com 30\% de substituição do cimento por metacaulim), no qual houve em média redução de $23 \%$ na resistência à compressão; entretanto, $10 \%$ de substituição (ensaio 9) nesse mesmo traço ocasionou aumento de 3\% na resistência aos 49 dias de cura.

Os resultados para os ensaios de absorção de água por imersão e a comparação deles com os resultados de referência estão representados esquematicamente na Figura 4.

Analisando os resultados apresentados na Figura 4, verifica-se que a adição de metacaulim não proporcionou alterações significativas na absorção de água por imersão, excetuando-se nos ensaios em que houve redução na resistência à compressão e, consequentemente, aumento na absorção de água. Entretanto, todos os resultados encontrados mantiveram-se abaixo de 6\%, estando de acordo, portanto, com as especificações da NBR 6136 (ABNT, 2007), a qual estabelece o limite de $10 \%$ para absorção em blocos vazados de concreto para alvenarias.

\begin{tabular}{|c|c|c|c|c|c|c|}
\hline \multirow{3}{*}{ Experimento } & \multicolumn{6}{|c|}{ Repetições } \\
\hline & \multicolumn{2}{|l|}{1} & \multicolumn{2}{|c|}{2} & \multicolumn{2}{|l|}{$\mathbf{3}$} \\
\hline & RC (MPa) & AA (\%) & RC (MPa) & AA (\%) & RC (MPa) & AA (\%) \\
\hline 1 & 17,81 & 5,56 & 18,01 & 5,28 & 18,02 & 5,17 \\
\hline 2 & 17,07 & 5,08 & 17,13 & 4,74 & 16,76 & 5,00 \\
\hline 3 & 18,34 & 4,83 & 18,06 & 4,52 & 18,16 & 4,23 \\
\hline 4 & 18,44 & 4,22 & 15,70 & 4,43 & 18,02 & 4,73 \\
\hline 5 & 23,00 & 4,25 & 22,38 & 4,06 & 20,56 & 4,18 \\
\hline 6 & 23,00 & 5,15 & 24,18 & 5,25 & 21,40 & 4,87 \\
\hline 7 & 25,57 & 4,40 & 22,06 & 4,18 & 21,67 & 4,11 \\
\hline 8 & 21,95 & 4,92 & 21,61 & 4,74 & 19,92 & 4,48 \\
\hline 9 & 22,61 & 5,07 & 21,41 & 5,05 & 22,21 & 5,02 \\
\hline 10 & 16,96 & 5,61 & 16,51 & 5,68 & 16,15 & 5,40 \\
\hline 11 & 18,20 & 4,12 & 17,13 & 4,22 & 16,01 & 4,04 \\
\hline 12 & 18,04 & 4,39 & 20,49 & 4,23 & 17,66 & 4,17 \\
\hline 13 & 15,44 & 5,85 & 14,89 & 5,74 & 14,81 & 5,45 \\
\hline 14 & 21,10 & 3,91 & 23,71 & 4,05 & 20,64 & 3,73 \\
\hline 15 & 22,13 & 4,09 & 20,79 & 3,92 & 20,60 & 4,06 \\
\hline 16 & 19,93 & 4,14 & 19,47 & 4,10 & 19,58 & 4,14 \\
\hline 17 & 20,79 & 3,82 & 22,80 & 3,98 & 22,32 & 3,94 \\
\hline
\end{tabular}

140 Rezende, M. L. de S.; Nascimento, J. W. B. do; Neves, G. de A.; Silva, F. L. H. da; Leal, A. F.; Ferreira, H. C. 


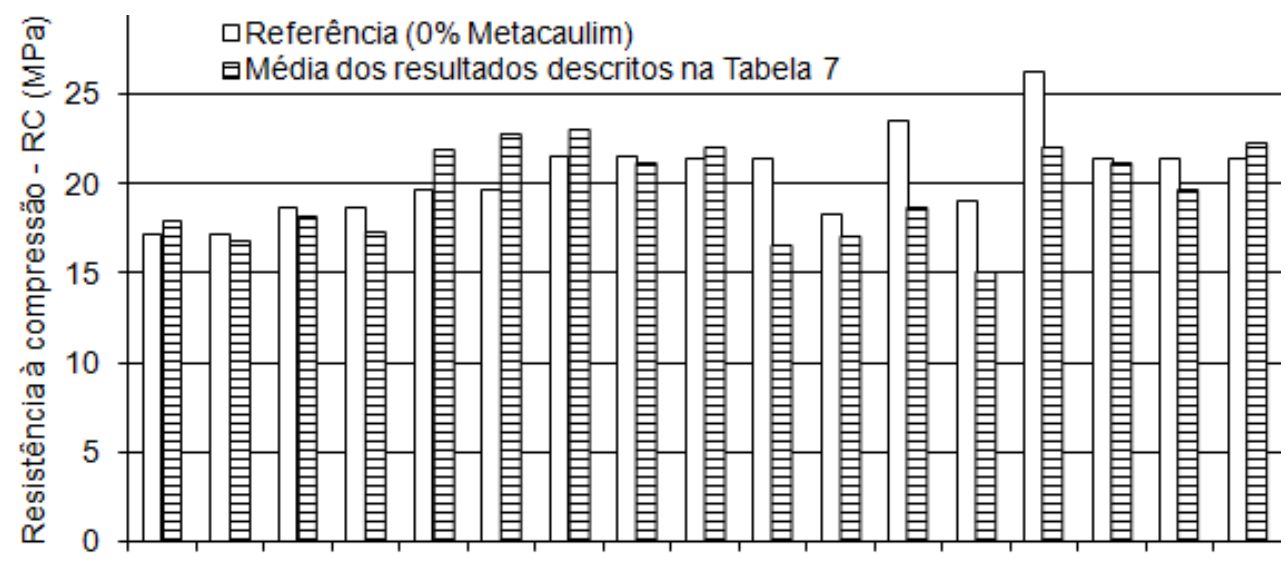

Figura 3 - Resultados para os ensaios de resistência à compressão (Tabela 7) comparados aos de referência $(0 \%$ de metacaulim)

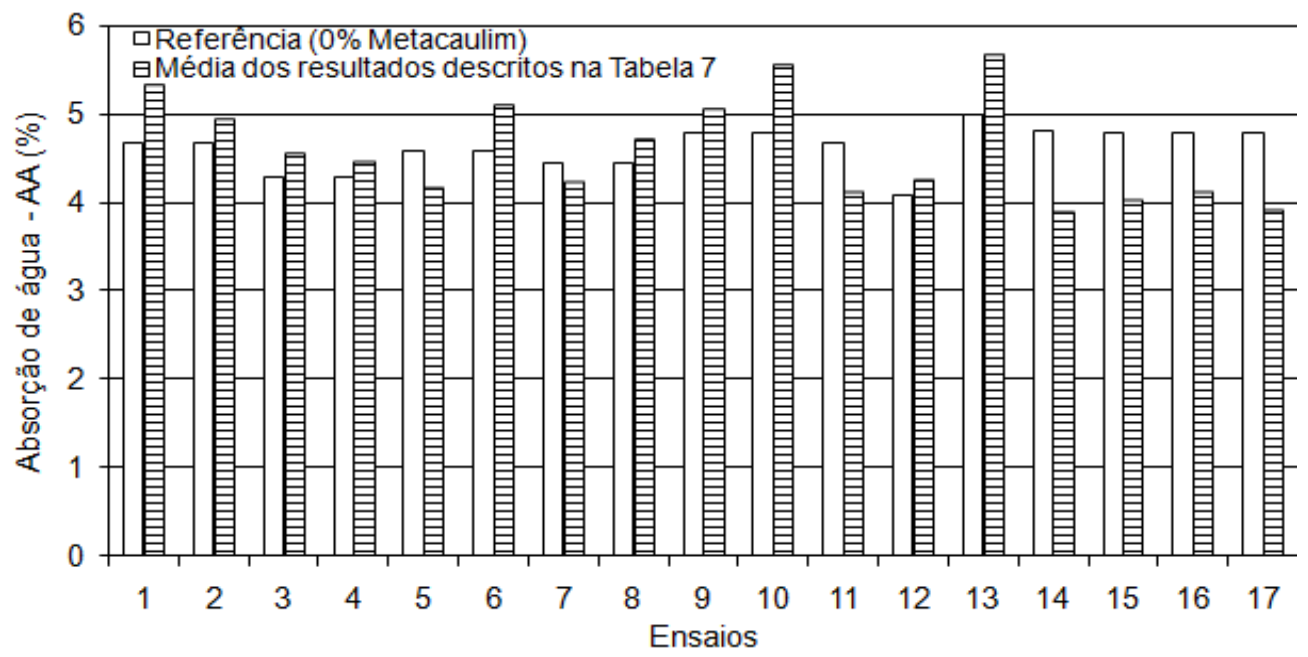

Figura 4 - Resultados para os ensaios de absorção de água (Tabela 7) comparados aos de referência (0\% de metacaulim)

As equações de regressão, estatisticamente significativas em um nível de 95\% de confiança, para as variáveis de resposta da resistência à compressão simples (RC) e absorção de água por imersão (AA), com os coeficientes estatisticamente significativos, são as seguintes:

$R C=20,84-0,89 T S+2,20 T C-0,72 C^{2}-$ $0,54 T C^{2}$ Eq. 1

$A A=4,03+0,13 T S-0,10 C-0,30 T C+$ $0,23 T S . T C+0,12 C . T C+0,43 T S^{2}+0,24 T C^{2}$

\section{Eq. 2}

Onde:

$T C=$ teor de cimento;

$T S$ = teor de substituição do cimento por metacaulim; e

$C=$ tempo de cura.
Os coeficientes de determinação $\left(\mathrm{R}^{2}\right)$, medida estatística de quanto uma linha de regressão aproxima-se de dados reais, para as Equações 1 e 2 foram de 0,73 e 0,79 respectivamente.

Os diagramas de Pareto para as duas variáveis de resposta (RC e AA) (Figuras 5a e 5b) permitem determinar quais coeficientes dos modelos realmente apresentam influência estatística ao nível de significância de 5\%.

Observando-se os gráficos da Figura 5, pode-se concluir que o coeficiente do modelo de maior relevância para a resistência à compressão é o teor de cimento, seguido do teor de substituição, para o coeficiente linear. Para a absorção de água, o coeficiente do modelo que apresenta maior influência é o teor de substituição quadrático. Observa-se também na Figura $5 b$ que todos os coeficientes do modelo são relevantes, tanto os 
lineares quanto os quadráticos, excetuando-se o coeficiente quadrático para o tempo de cura e a interação teor de substituição e tempo de cura.

Na Tabela 8 constam os dados resultantes da análise de variância (ANOVA), teste $\mathrm{F}$ e coeficientes de determinação $\left(\mathrm{R}^{2}\right)$.

Pode-se concluir, observando os dados da Tabela 8 , que, apesar de os coeficientes de determinação $\left(\mathrm{R}^{2}\right)$ apresentarem baixos valores, o modelo pode ser considerado estatisticamente significativo. Isso é demonstrado pelo fato de que os valores calculados para $\mathrm{F}$ foram acima de 6 vezes o valor do Ftabelado (RODRIGUES; IEMMA, 2009).

O uso do método de análise por superfície de resposta (MSR) permite estimar os níveis mais representativos para os resultados esperados. Têmse como valores ideais para as variáveis independentes em estudo os que possam ser produzidos com maior teor de metacaulim e resultem em maior resistência à compressão e menor índice de absorção de água, considerandose o menor consumo de cimento e o menor tempo de cura.

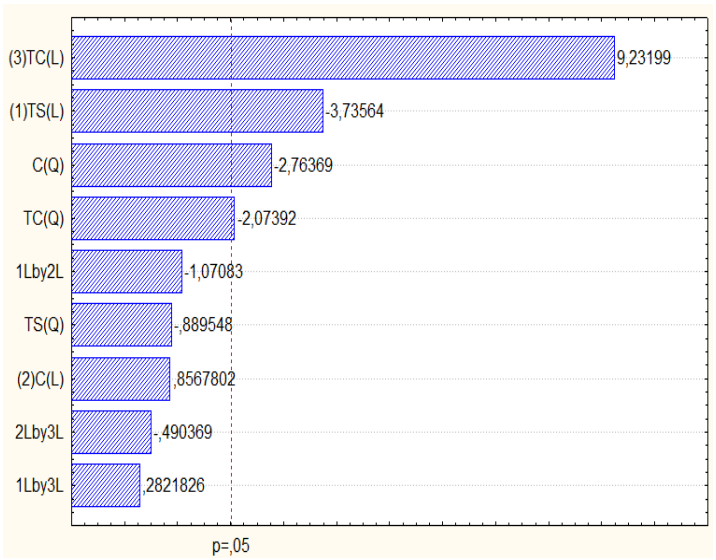

(a)
As superfícies de resposta geradas a partir da análise estatística dos resultados para os ensaios de resistência à compressão simples, em função do teor de substituição do cimento por metacaulim e o tempo de cura, utilizando-se o programa Statistica 5.0 da Statsoft, estão apresentadas na Figura 6.

Nota-se, observando as curvas representadas na Figura 6, que as resistências tendem a reduzir-se com o aumento da substituição do cimento por metacaulim; entretanto, em todos os traços verifica-se que os melhores resultados são alcançados no nível 0 (ponto central, média aritmética dos níveis -1 e +1 ) para o tempo de cura, ou seja, em média, 49 dias, que estão associados aos níveis $-1,68$ e 0 para os teores de substituição, ou seja, $10 \%$ e $20 \%$ respectivamente. Tais resultados estão de acordo com os encontrados por Cassagnabère et al. (2009), os quais encontraram os melhores resultados para resistência à compressão simples com teores de substituição da massa do cimento por metacaulim variando entre $12,5 \%$ e $25 \%$.

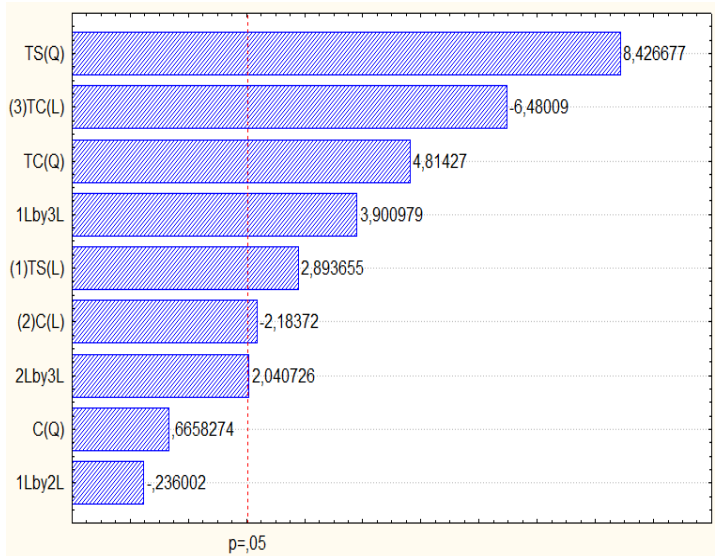

(b)

Figura 5 - Diagrama de Pareto para a (a) resistência à compressão simples e (b) absorção de água por imersão

Nota: Legenda:

TC: Teor de cimento;

TS: Teor de substituição do cimento por metacaulim; e

C: Tempo de cura.

Tabela 8 - Análise de variância para avaliação do uso do metacaulim em concreto seco

\begin{tabular}{llrrrrrr}
\hline $\begin{array}{l}\text { Propriedade } \\
\text { avaliada }\end{array}$ & & SQ & GL & QM & F $_{\text {calculado }}$ & F $_{\text {tabelado }}$ & $\mathbf{R}^{\mathbf{2}}$ \\
\hline Resistência à & Regressão & 256,6566 & 9 & 28,5174 & 12,31 & 2,21 & 0,73 \\
compressão & Resíduo & 94,9648 & 41 & 2,3162 & & & \\
simples & Total & 351,6214 & 50 & & & & \\
\hline Absorção de & Regressão & 13,6555 & 9 & 1,5173 & 17,54 & 2,21 & 0,79 \\
água por & Resíduo & 3,5457 & 41 & 0,0865 & & & \\
imersão & Total & 17,2012 & 50 & & & & \\
\hline
\end{tabular}

142 Rezende, M. L. de S.; Nascimento, J. W. B. do; Neves, G. de A.; Silva, F. L. H. da; Leal, A. F.; Ferreira, H. C. 


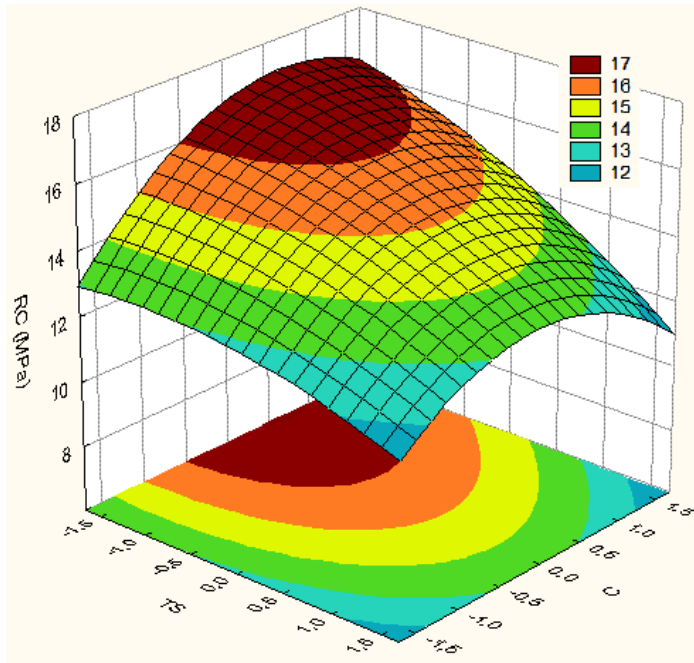

(a) Traço 1:7,67 (N-1,68)

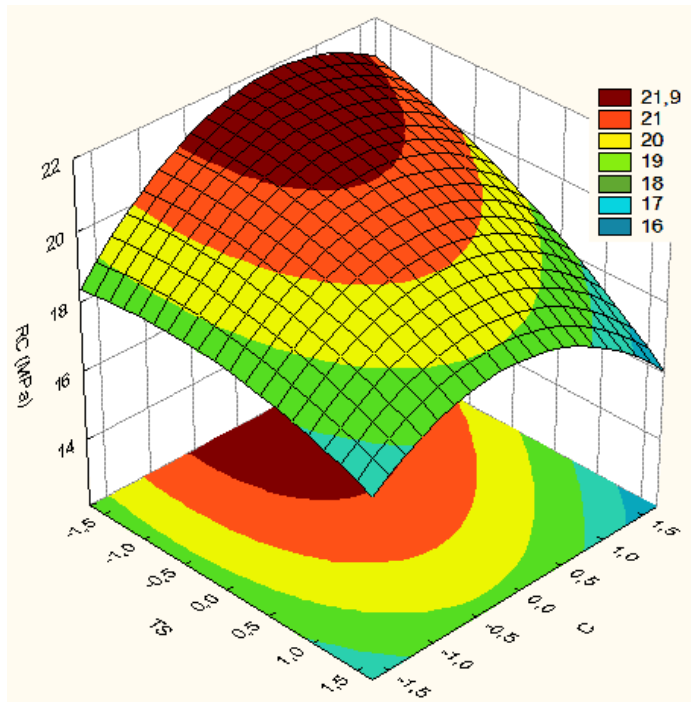

(c) Traço 1:5,15 (N 0)

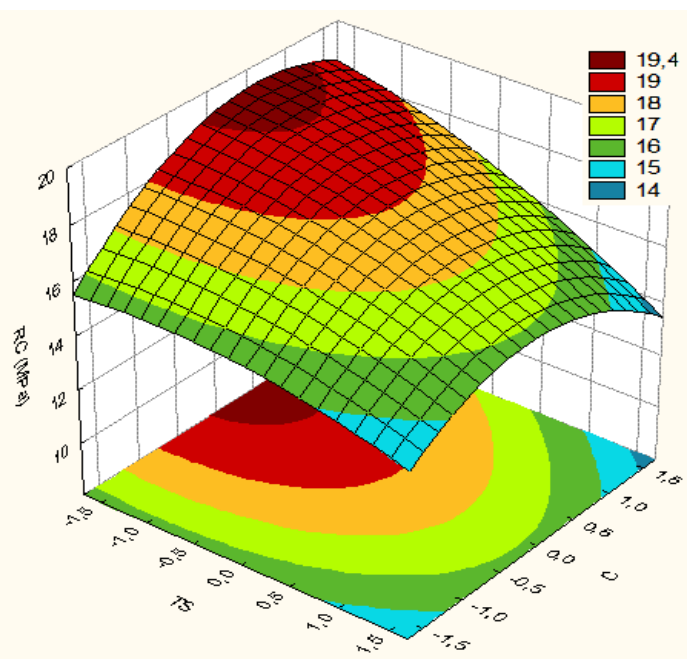

(b) Traço 1:6,44 (N -1)

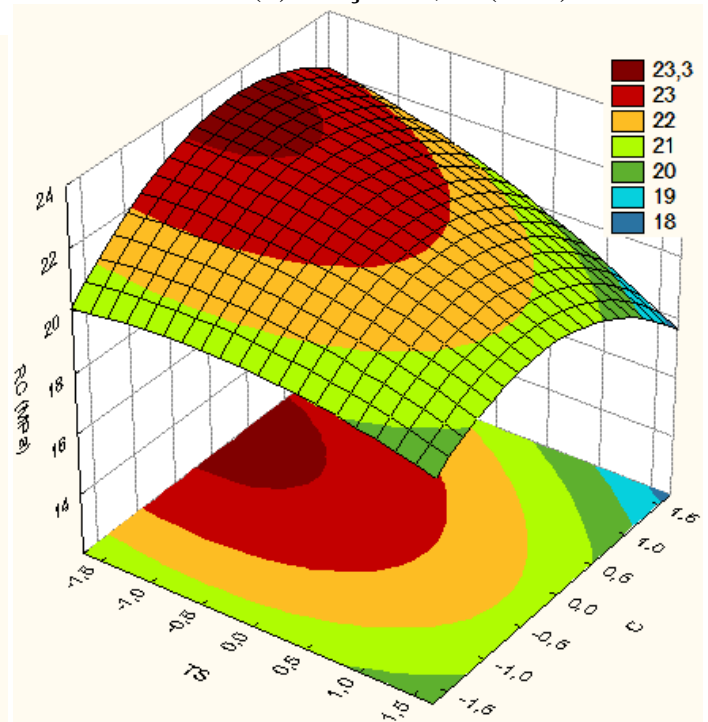

(d) Traço 1:4,25 (N+1)

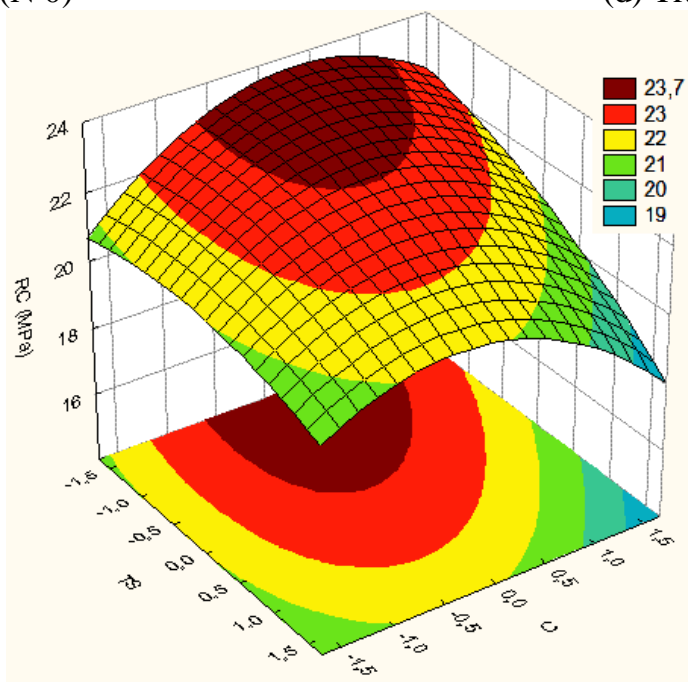

(e) Traço 1:3,83 $(\mathrm{N}+1,68)$

Figura 6 - Superfícies de resposta para a resistência à compressão, em função do teor de substituição do cimento por metacaulim e do tempo de cura, para os cinco traços avaliados 
As superfícies de resposta para os ensaios de absorção de água por imersão, em função do teor de substituição do cimento por metacaulim e o tempo de cura, estão apresentadas na Figura 7.

Analisando as Figuras 7a, 7b, 7c, 7d e 7e, nota-se que os valores para absorção de água por imersão tendem a reduzir com o tempo de cura e tendem a

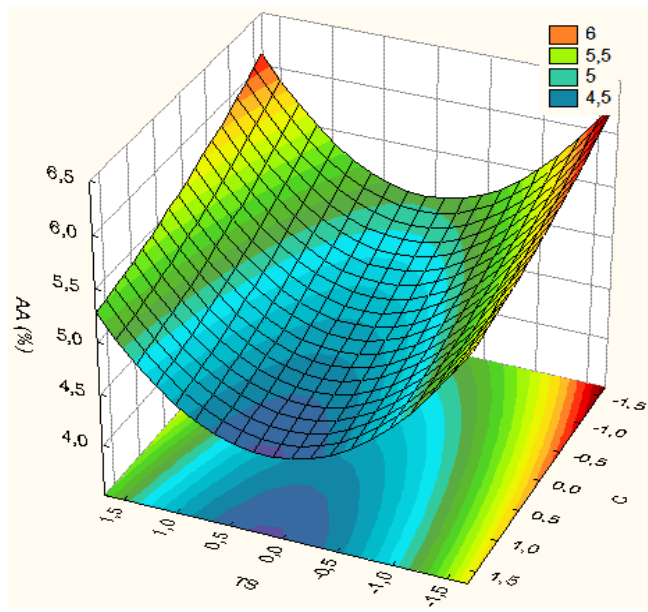

(a) Traço 1:7,67 (N -1,68)

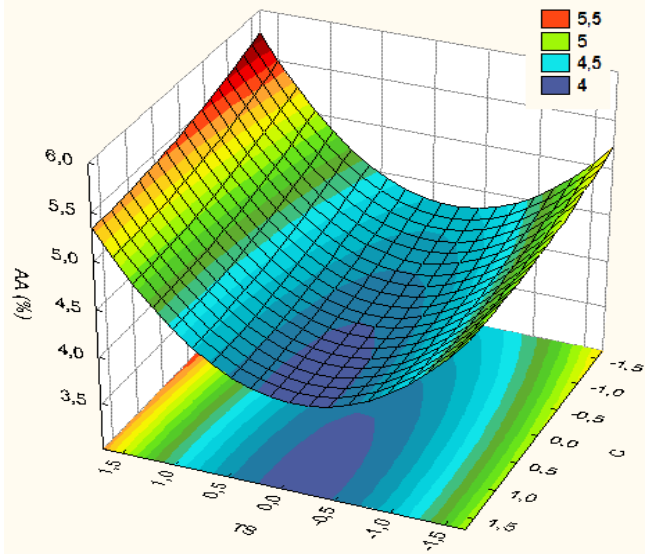

(c) Traço 1:5,15 (N 0) elevar-se com o aumento do teor de metacaulim. Entretanto, verifica-se que, para todos os traços, o teor de substituição que resulta em menores índices de absorção é o nível 0 (ponto central), ou seja, $20 \%$ de substituição do cimento por metacaulim.

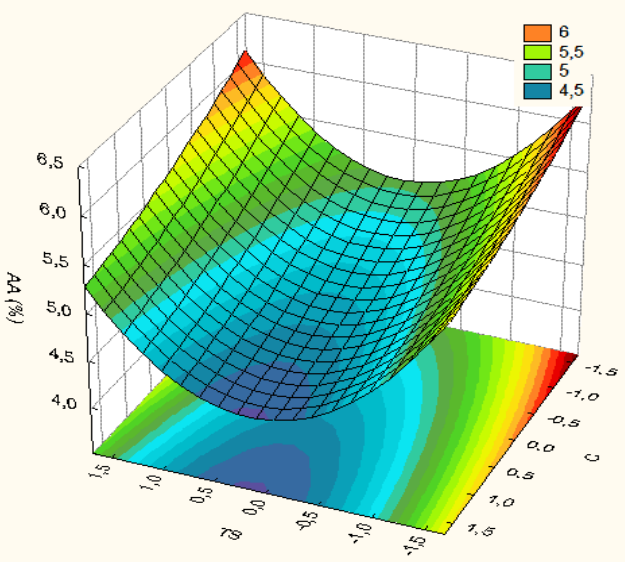

(b) Traço 1:6,44 (N -1)

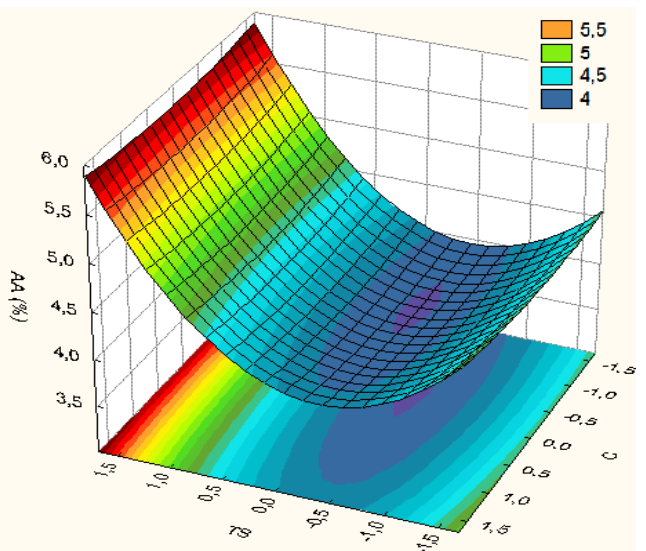

(d) Traço 1:4,25 ( $\mathrm{N}+1)$

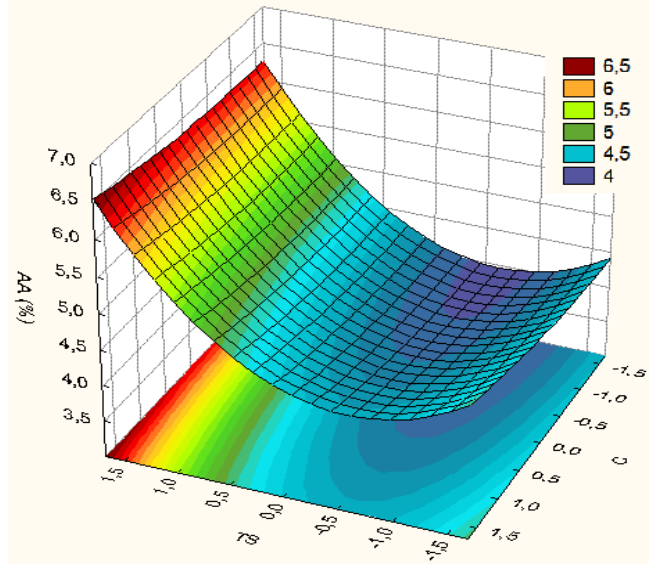

(e) Traço 1:3,83 $(\mathrm{N}+1,68)$

Figura 7 - Superfícies de resposta para a absorção de água por imersão nos cinco traços avaliados 


\section{Conclusões}

A metodologia de superfície de resposta mostrouse eficiente para os objetivos propostos neste trabalho, permitindo estimar os níveis de substituição da massa do cimento por metacaulim nos cinco traços estudados. Para todos os traços, pode-se concluir que o uso de metacaulim em percentuais variando entre $10 \%$ e $20 \%$ da massa do cimento poderá promover aumento na resistência à compressão e redução na absorção de água por imersão. Entretanto, tais resultados servem apenas de referência para futuras pesquisas, devendo ser investigados por meio de análises térmicas e de difração de raios $\mathrm{X}$ para avaliação do consumo de hidróxido de cálcio livre e formação de silicatos de cálcio hidratados, comprovando, assim, a atividade pozolânica.

\section{Referências}

ASSOCIAÇÃO BRASILEIRA DE NORMAS TÉCNICAS. NBR 5733 EB 2: cimento portland de alta resistência inicial. Rio de Janeiro, 1991a.

ASSOCIAÇÃO BRASILEIRA DE NORMAS TÉCNICAS. NBR 6136: blocos vazados de concreto simples para alvenaria: requisitos. Rio de Janeiro, 2007.

ASSOCIAÇÃO BRASILEIRA DE NORMAS TÉCNICAS. NBR 7211: agregados para concreto: especificação. Rio de Janeiro, 2009.

ASSOCIAÇÃO BRASILEIRA DE NORMAS TÉCNICAS. NBR 7215: cimento portland: determinação da resistência à compressão. Rio de Janeiro, 1996.

ASSOCIAÇÃO BRASILEIRA DE NORMAS TÉCNICAS. NBR 11579: cimento portland: determinação da finura por meio da peneira $75 \mu \mathrm{m}$ $\left(n^{\circ} 200\right)$. Rio de Janeiro, 1991b.

\section{ASSOCIAÇÃO BRASILEIRA DE NORMAS}

TÉCNICAS. NBR 12118: blocos vazados de concreto simples para alvenaria: métodos de ensaio. Rio de Janeiro, 2010.

ASSOCIAÇÃO BRASILEIRA DE NORMAS TÉCNICAS. NBR NM 23: cimento portland e outros materiais em pó: determinação da massa específica. Rio de Janeiro, 2000.

ASSOCIAÇÃO BRASILEIRA DE NORMAS TÉCNICAS. NBR NM 45: agregado em estado solto: determinação da massa unitária. Rio de Janeiro, 2006.
ASSOCIAÇÃO BRASILEIRA DE NORMAS

TÉCNICAS. NBR NM 53: agregados:

determinação da absorção e da massa específica do agregado graúdo: método de ensaio. Rio de Janeiro, 2003a.

ASSOCIAÇÃO BRASILEIRA DE NORMAS TÉCNICAS. NBR NM 65: cimento portland: determinação do tempo de pega. Rio de Janeiro, 2002.

\section{ASSOCIAÇÃO BRASILEIRA DE NORMAS}

TÉCNICAS. NBR NM 248: agregados: determinação da composição granulométrica. Rio de Janeiro, 2003b.

AL-AKHRAS, N. M. Durability of Metakaolin Concrete to Sulfate Attack. Cement and Concrete Research, v. 36, n. 9, p. 1727-1734, 2006.

CARMO, J. B. M.; PORTELLA, K. F. Estudo Comparativo do Desempenho Mecânico da Sílica Ativa e do Metacaulim Como Adições Químicas Minerais em Estruturas de Concreto. Cerâmica, São Paulo, v. 54, n. 331, p. 309-318, set. 2008.

CASSAGNABÈRE, F. et al. Use of Flash Metakaolin in a Slip-Forming Concrete For the Precast Industry. Magazine of Concrete Research, v. 61, n. 10, p. 767-778, dez. 2009.

FERNANDES, I. D. Blocos e Pavers: produção e controle de qualidade. Ribeirão Preto, SP: Treino Assessoria e Treinamentos Empresariais Ltda., 2008. 120 p.

FRASSON JUNIOR, A. Proposta de Metodologia de Dosagem e Controle do Processo Produtivo de Blocos de Concreto Para Alvenaria Estrutural. 162 f. Florianópolis, 2000. Dissertação (Mestrado em Engenharia Civil) Escola de Engenharia, Universidade Federal de Santa Catarina, Florianópolis, 2000.

GRUBER, K. A. et al. Increasing Concrete Durability With High-Reactivity Metakaolin. Cement and Concrete Composites, v. 23, n. 6, p. 479-484, 2001.

GUNAWAN, E. R. et al. Study on Response Surface Methodology (RSM) of Lipase-Catalyzed Synthesis of Palm-Based Wax Esters. Enzyme and Microbial Technology, v. 37, n. 7, p. 739744, 2005.

PETTERMANN, R. Avaliação do Desempenho de Blocos de Concreto Para Pavimentação Com Metacaulim e Sílica Ativa. 71 f. Porto Alegre, 2006. Monografia (Especialização em Construção Civil) -Escola de Engenharia Civil, Universidade Federal do Rio Grande do Sul, Porto Alegre, 2006. 
RODRIGUES, M. I.; IEMMA, A. F. Planejamento de Experimentos \& Otimização de Processos. 2. ed. revisada e ampliada. Campinas, SP: Casa do Pão, 2009. p. 125-133.

ROSSIGNOLO, J. A.; OLIVEIRA, I. L. Concreto Leve Estrutural Com Metacaulim. Minerva, v. 3, n. 2, p. 177-187, 2006.

SABIR, B. B.; WILD, S.; BAI, J. Metakaolin and Calcined Clays as Pozolans For Concrete: a review. Cement and Concrete Composites, v. 23, n. 2, p. 441-454, 2001.

SIDDIQUE, R.; KLAUS, J. Influence of Metakaolin on the Properties of Mortar and Concrete: a review. Applied Clay Science, v. 43, n. 3/4, p. 392-400, mar. 2009.

SINDICATO NACIONAL DA INDÚSTRIA DE PRODUTOS DE CIMENTO. Índices

SINAPROCIM Janeiro a Dezembro de 2010. Disponível em: <www.sinaprocim.org.br>. Acesso em: 12 dez. 2011.
SOUZA, P. S. L.; DAL MOLIN, D. C. C. Estudo da Viabilidade do Uso de Argilas Calcinadas, Como Metacaulim de Alta Reatividade (MCAR). Engenharia Civil-UM, v. 15, p. 45-54, set. 2002.

SOUZA, P. S. L. Verificação da Influência do Uso de Metacaulim de Alta Reatividade nas Propriedades Mecânicas do Concreto de Alta Resistência. 203 f. Porto Alegre, 2003. Tese (Doutorado em Engenharia Civil) - Escola de Engenharia, Universidade Federal do Rio Grande do Sul, Porto Alegre, 2003.

VU, D. D.; STROEVEN, P. BUI, V. B. Strength and Durability Aspects of Calcined KaolinBlended Portland Cement Mortar and Concrete. Cement and Concrete Composites, v. 23, n. 6, p. 471-478, 2001.

\section{Agradecimentos}

À empresa de elementos pré-moldados JAR Indústria de Premoldados Ltda., pelo fornecimento do material utilizado nesta pesquisa.

Revista Ambiente Construído

Associação Nacional de Tecnologia do Ambiente Construído

Av. Osvaldo Aranha, 99 - 3o andar, Centro

Porto Alegre - RS - Brasil CEP $90035-190$

Telefone: +55 (51) 3308-4084

Fax: +55 (51) 3308-4054

www. seer. ufrgs. br/ ambienteconstruido

E-mail: ambienteconstruido@ufrgs.br

146 Rezende, M. L. de S.; Nascimento, J. W. B. do; Neves, G. de A.; Silva, F. L. H. da; Leal, A. F.; Ferreira, H. C. 